\title{
BODIES FOR WHICH HARMONIC FUNCTIONS SATISFY THE MEAN VALUE PROPERTY( $\left.{ }^{1}\right)$
}

\author{
BY \\ AVNER FRIEDMAN AND WALTER LITTMAN
}

Introduction. Let $D$ be an $n$-dimensional domain with boundary $S$, let $\mu$ be a density function defined on $S$, and let $P$ be a fixed point in $D$. As is well known, if $S$ is a sphere and $P$ is its center, then for any harmonic function in $D$, continuous in $D+S$,

$$
u(P)=\int_{S} u(Q) \mu(Q) d S_{Q},
$$

where $\mu(Q) \equiv$ const depending on $n$. In this paper we consider the converse problem, namely: Suppose (0.1) holds for all harmonic functions, is $S$ necessarily a sphere with center $P$ (and consequently $\mu \equiv$ const)? The answer is in general negative, unless some assumptions are made on $\mu$. Thus, $(0.1)$ is always satisfied with $\mu(Q)=-\partial G(P, Q) / \partial \nu, G$ being Green's function of the Laplace equation in $D$ and $\nu$ being the outward normal to $S$.

Denoting by $A$ and $B$ any two points on $S$ which are furthest and nearest to $P$ respectively, we prove (in $\S \S 1,2$ ) that if $\mu(A) \geqq \mu(B)$ then $S$ is necessarily $a$ sphere with center $P$. Some differentiability assumptions are made on the behavior of $S$ in some neighborhoods of the points $A, B$.

The condition $\mu(A) \geqq \mu(B)$ is a sharp condition, and this fact is used (in $\S 3$ ) to derive an application (of $\$ \S 1,2$ ) to conformal mappings.

We further consider (in \$4) an analogous mean value problem with respect to solids, namely, if

$$
u(P)=\int_{D} u(Q) \mu(Q) d V_{Q}
$$

holds for all harmonic functions, is $S$ necessarily a sphere with center $P$ ? The answer is in the affirmative, provided (again) $\mu$ satisfies some conditions (which, this time, involve the knowledge of $\mu$ in the whole domain $D$ ). The special case where $D$ is convex, $\mu \equiv$ const and $n=2$ was previously solved by Brödel [0]. His method, which is entirely different from ours, can easily be extended to the case $n>2$, but it cannot be extended to general $D$ and $\mu$.

In $\$ 5$ we generalize the results of $\S \S 1,2,4$ to the case of solutions of the heat equation. The outline of the results and methods is analogous to the harmonic case, but new technical ideas are employed.

Received by the editors April 3, 1961.

(1) Prepared under Contract Nonr 710(16)(NR 044 004) between the Office of Naval Research and the University of Minnesota. 
Finally, in $\S 6$, we briefly mention some typical situations arising in the case of solutions of the wave equation.

1. Mean values on hypersurfaces. Let $D$ be an $n$-dimensional bounded domain and denote its boundary by $S$. We fix a point $P$ in $D$ and a function $\mu$ on $S$ and ask: Under what conditions does the mean value property

$$
u(P)=\int_{S} u(Q) \mu(Q) d S_{Q}
$$

hold for all harmonic functions? In this and in the following section we give some sufficient conditions on $S$ and $\mu$ which imply that $S$ is necessarily a sphere with center $P$. In order to state these conditions, we introduce some notations.

By a differentiability class $C^{m}$ we mean: the first $[m]$ derivatives satisfy a Hölder condition of exponent $m-[m]$ if $m>[m]$, and are uniformly continuous if $m=[m]$. We say that $S$ is piecewise $C^{m}$ if $S$ is an $n-1$ manifold and if it is a union of a finite number of disjoint sets $S=\sum S_{i}+\sum \sum S_{i j}$ where the $S_{i}$ are $(n-1)$-dimensional $C^{m}$ (in the local parameters) open hypersurfaces and the $S_{i j}$ are $j$-dimensional continuously differentiable surfaces, $1 \leqq j \leqq n-2$; $S_{i 0}$ are isolated points. Throughout this paper $S$ is always assumed to be at least piecewise $C^{1}$, and $D$ is always assumed to be bounded.

We fix points $A, B$ on $S$ which satisfy:

$$
\max _{Q \in S} d(P, Q)=d(P, A), \quad \min _{Q \in S} d(P, Q)=d(P, B),
$$

where $d(P, Q) \equiv r_{P Q}$ is the Euclidean distance from $P$ to $Q . D$ is called a Dirichlet domain if the Dirichlet problem, for Laplace's equation, has a solution in $D$ for any continuous boundary values.

We can now state the main result of the present section.

THEOREM 1. Let $\mu$ be an (absolutely) integrable function on $S$, continuous at the points $A, B$, and let $\mu(A) \geqq \mu(B)$. Let $D$ be a Dirichlet domain $\left({ }^{2}\right)$ and let $S$ be $C^{1+\alpha}$ (for some $\alpha>0$ ) in some neighborhoods of $A, B$. If the mean value property (1.1) holds for all harmonic polynomials, then $S$ is a sphere with center $P$.

Proof. We first claim that (1.1) holds also for any function $u$ harmonic in $D$ and continuous in $D+S$. Indeed, let $D_{\epsilon}$ be any smooth domain containing $D$ and contained in an $\epsilon$-neighborhood of $D$. As is well known, any harmonic function $u_{\epsilon}$ in $D_{\epsilon}$ can be approximated, uniformly in $D+S$, by a sequence of harmonic polynomials $\left({ }^{3}\right)$. Applying (1.1) to each member of the sequence we conclude that (1.1) also holds for $u_{\epsilon}$. Taking now an appropriate sequence of

(2) Added in proof: Strictly speaking, we must assume that the closure of $D$ has connected complement. If this is not the case, then the assertion of Theorem 1 remains true if we assume that (1.1) holds for any function harmonic in some neighborhood of $D$. A similar remark applies also to Theorems $2,4,5$ below.

(3) See [16]. 
domains $D_{\epsilon}\left(D_{\epsilon} \rightarrow D\right.$ as $\left.\epsilon \rightarrow 0\right)$ with boundary values converging uniformly to those of $u$ on $S$, and applying (1.1) to each $u_{\epsilon}$, we conclude that (1.1) holds also for $u$.

We next introduce the Green's function $G(P, Q)$ of the Laplace equation in $D$, having its singularity at $P . G(P, Q)$ is a continuous function in $Q \in D+S$ and it vanishes on $S$. Let $S_{0}$ be any open portion of $S$ which is $C^{1+\alpha}$. By a theorem of Kellogg [6], $G$ is continuously differentiable on $D+S_{0}$. We shall need the following lemma:

LEMMA 1. If $u$ is harmonic in $D$, continuous in $D+S$ and vanishes outside a closed portion $S_{1} \subset S_{0}$, then

$$
u(P)=-\int_{S_{1}} \frac{\partial G(P, Q)}{\partial \nu} u(Q) d S_{Q}
$$

where $\nu$ is the outward normal to $S($ at $Q)$.

Proof. We first prove the lemma under the additional assumptions that $u$ is non-negative and continuously differentiable in $D+S_{0}$.

Consider the manifolds $G(P, Q)=\epsilon$. As was proved by Kellogg $[5 ; 7$, p. 277] the gradient of any harmonic function in $D$ vanishes at most on a set of points lying on a denumerable number of equipotential hypersurfaces. Hence, we can always take an appropriate sequence of $\epsilon \rightarrow 0$, such that the hypersurfaces $G(P, Q)=\epsilon$ are (in some local parameters) continuously differentiable (and, in fact, analytic). The set $D^{\epsilon}$ defined by $G>\epsilon$ is a domain [7, pp. 238-239], and if its boundary $S^{\epsilon}$ is continuously differentiable, then the Divergence Theorem can be used in the domain. Hence, we obtain Green's formula

$$
u(P)=-\int_{S^{\epsilon}} u(Q) \frac{\partial G(P, Q)}{\partial \nu} d S_{Q}^{\epsilon}+\int_{S^{\epsilon}} G(P, Q) \frac{\partial u(Q)}{\partial \nu} d S_{Q}^{\epsilon}
$$

where $\nu$ is the outward normal to $S^{\epsilon}$.

On $S^{\epsilon}, G=\epsilon$. Hence the second integral on the right side of (1.3) is zero. To consider the first integral, we first make the assumption that the diameter of the set $S_{1}$ is sufficiently smali. We construct the tangent hyperplane to $S$ at a point $Q_{0}$ on $S_{1}$ and denote by $T_{1}$ the orthogonal projection of $S_{1}$ on the hyperplane. Let $S_{2}$ be a closed portion of $S_{0}$ which contains $S_{1}$ and denote by $T_{2}$ its projection on the tangent hyperplane. Now let $T$ be a domain with continuously differentiable boundary, on the tangent hyperplane, satisfying $T_{1} \subset T \subset T_{2}$.

We next construct a right cylinder with base $T$. It intersects $S$ by $\hat{S}$, $S^{\boldsymbol{\epsilon}}$ by $\hat{S}^{\boldsymbol{\epsilon}}$ and $D-D^{\boldsymbol{\epsilon}}$ by a set $V^{\boldsymbol{\epsilon}}$. Let $L^{\boldsymbol{\epsilon}}$ be the part of the boundary of $V^{\boldsymbol{\epsilon}}$ lying on the lateral boundary of the cylinder $\left.{ }^{4}\right)$. If the diameter of $S_{1}$ is sufficiently

(4) To be more precise, $V^{\epsilon}$ is that component of what is defined above as $V^{\epsilon}$, which touches Q. Also we restrict $\hat{S}, \hat{S}^{\epsilon}$ and $L^{\epsilon}$ accordingly. 
small, then $V^{\epsilon}$ is a domain in which the Divergence Theorem holds. Hence we get, using Green's identity,

$$
\begin{aligned}
\int_{\hat{S}} u \frac{\partial G}{\partial \nu} d S-\int_{\hat{S}^{e}} u \frac{\partial G}{\partial \nu} d S= & \int_{L^{e}}\left(G \frac{\partial u}{\partial \nu}-u \frac{\partial G}{\partial \nu}\right) d S \\
& +\int_{\hat{S}} G \frac{\partial u}{\partial \nu} d S-\int_{\hat{S}^{e}} G \frac{\partial u}{\partial \nu} d S
\end{aligned}
$$

where $\nu$ on $L^{\epsilon}+\hat{S}$ points out of $V^{\epsilon}$, whereas $\nu$ on $\hat{S}^{\epsilon}$ points into $V^{\epsilon}$. In writing down (1.4), we used the fact that $u$ is continuously differentiable in $D+S_{0}$.

Now the second integral on the right side of (1.4) vanishes. The third integral does not exceed $\epsilon \int|\partial u / \partial \nu| d S$; hence it tends to zero as $\epsilon \rightarrow 0$. Clearly, the first integral on the right side of (1.4) also tends to zero as $\epsilon \rightarrow 0$. We conclude that

$$
\int_{\hat{S}^{e}} u \frac{\partial G}{\partial \nu} d S \rightarrow \int_{\hat{S}} u \frac{\partial G}{\partial \nu} d S
$$

Since $u(Q) \rightarrow 0$ as $Q$ approaches $S-S_{1}$, and since $u \geqq 0$ in $D$ and $\partial G / \partial \nu \leqq 0$ on $S^{\ddagger}$, we have

$$
0 \leqq-\int_{S^{\epsilon}-\hat{S}^{\epsilon}} u \frac{\partial G}{\partial \nu} d S \leqq \max _{S^{\epsilon}-\hat{S}^{\epsilon}} u(R) \cdot\left(-\int_{S^{\epsilon}} \frac{\partial G}{\partial \nu} d S\right) \rightarrow 0
$$

as $\epsilon \rightarrow 0$ (recalling that $-\int_{S^{\epsilon}}(\partial G / \partial \nu) d S=1$ ).

Combining (1.5), (1.6) and taking $\epsilon \rightarrow 0$ in (1.3), we obtain (1.2).

Having proved (1.2) for $S_{1}$ with a small diameter, the case of arbitrary $S_{1}$ follows by superposition, using partition of unity to break up the non-negative and differentiable boundary value of $u$ into a finite number of non-negative differentiable functions, each having a support on a $C^{1+\alpha}$ portion with a sufficiently small radius.

Having established (1.2) for non-negative and continuously differentiable functions $u$ (in $D+S_{0}$ ) we next extend it to non-negative functions which are only continuous in $D+S$, by approximating the non-negative boundary values by non-negative and continuously differentiable boundary values vanishing outside $\eta$-neighborhoods of $S_{1}$ on $S$, with $\eta \rightarrow 0$. Finally, in order to establish (1.2) for $u$ which is not necessarily non-negative, we break the boundary values of $u$ on $S_{1}$ into a difference of two non-negative functions, solve the Dirichlet problem for each of the two boundary functions, and apply (1.2) to each of them. The proof of Lemma 1 is thus completed.

Comparing (1.1), (1.2) we get:

Lemma 2. On a portion $S_{0}$ of $S$ which is $C^{1+\alpha}$,

$$
-\frac{\partial G(P, Q)}{\partial \nu}=\mu(Q)
$$


almost everywhere. In particular, if $\mu$ is continuous at a point $Q$ of $S_{0}$, then (1.7) holds at $Q$.

We proceed to prove Theorem 1. Let $K_{A}$ and $K_{B}$ be balls with center $P$ and radii $d(P, A)$ and $d(P, B)$ respectively. Denote the corresponding boundaries by $S_{A}$ and $S_{B}$. We compare Green's functions $G_{A}(P, Q), G_{B}(P, Q)$ of $K_{A}, K_{B}$ with Green's function $G(P, Q)$ of $D$.

By the maximum principle we have $G_{A}>G$ on $D$. Since $G_{A}=G=0$ at $A$, we find that

$$
\frac{\partial G_{A}(P, Q)}{\partial \nu} \leqq \frac{\partial G(P, Q)}{\partial \nu} \quad \text { at } Q=A,
$$

where $\nu$ is the joint outward normal to $S$ and $S_{A}$ at the point $A$ (where the two hypersurfaces are tangent).

Similarly,

$$
\frac{\partial G_{B}(P, Q)}{\partial \nu} \geqq \frac{\partial G(P, Q)}{\partial \nu} \quad \text { at } Q=B .
$$

We next recall that, if $n>2$,

$$
G_{A}(P, Q)=\gamma_{n}\left(r_{P Q}^{2-n}-r_{P A}^{2-n}\right) \quad\left(\gamma_{n} \text { is some positive constant }\right)
$$

and similarly for $G_{B}(P, Q)$. Hence, if $r_{P A}>r_{P B}$,

$$
\text { (1.10) }\left.\frac{\partial G_{A}(P, Q)}{\partial r_{P Q}}\right|_{Q=A}=\gamma_{n}(2-n) r_{P A}^{1-n}>\gamma_{n}(2-n) r_{P B}^{1-n}=\left.\frac{\partial G_{B}(P, Q)}{\partial r_{P Q}}\right|_{Q-B} \text {. }
$$

Noting that the normal to $S$ at $A$ is orthogonal to $S_{A}$ so that $\partial / \partial \nu=\partial / \partial r_{P Q}$ for $Q=A$ (and similarly for $B$ ), we may combine (1.10) with (1.9), (1.8) and get

$$
\frac{\partial G(P, A)}{\partial \nu}>\frac{\partial G(P, B)}{\partial \nu}
$$

By our assumptions on $\mu$ and by Lemma 2 we conclude, however, that $\partial G(P, A) / \partial \nu \leqq \partial G(P, B) / \partial \nu$, and we have thus derived a contradiction, assuming $r_{P A}>r_{P B}$. Hence we must have $r_{P A}=r_{P B}$, i.e., $S$ is a sphere with radius $P$.

In the case $n=2$, we have to take

$$
G_{A}(P, Q)=\gamma_{2} \log \left(r_{P A} / r_{P Q}\right)
$$

and similarly for $G_{B}$.

REMARK 1 . Since on a sphere with center $P, \partial G / \partial \nu$ is constant, we may add to the assertions of Theorem 1 the assertion that $\mu=$ const almost everywhere. 
REMARK 2. The derivation of (1.11) can also be obtained in the following way: Consider the harmonic function

$$
h(Q)=G(P, Q)-\gamma_{n} r_{P Q}^{2-n} .
$$

It is clear that $h<0$ on $S$ and it attains its absolute maximum at $Q=A$ and its absolute minimum at $Q=B$. Recalling that $\partial / \partial \nu=\partial / \partial r_{P Q}$ at $Q=A$ and at $Q=B$, we obtain

$$
\begin{aligned}
& 0 \leqq \frac{\partial h(A)}{\partial \nu}=\frac{\partial G(P, A)}{\partial \nu}+\gamma_{n}(n-2) r_{P A}^{1-n}, \\
& 0 \geqq \frac{\partial h(B)}{\partial \nu}=\frac{\partial G(P, B)}{\partial \nu}+\gamma_{n}(n-2) r_{P A}^{1-n},
\end{aligned}
$$

from which (1.11) follows (if $r_{P A}>r_{P B}$ ).

The advantage of the first method is that it can be generalized to the case of the heat equation (see $\$ 5$ ).

REMARK 3. If we write (1.1) in the form

$$
u(0)=\int_{S} u(Q) \mu(Q) d S_{Q}
$$

where $P=0$ is the origin, then since $u(\lambda Q)$ ( $\lambda$ real) is also a harmonic polynomial, we conclude that (1.1) implies the apparently stronger property

$$
u(0)=\int_{S} u(\lambda Q) \mu(Q) d S_{Q} .
$$

REMARK 4. From the proof of Theorem 1 it follows that for any domain $D$ with boundary $C^{1+\alpha}$, the mean value property (1.1) holds with $\mu(Q)$ $=-\partial G(P, Q) / \partial \nu$. Also, at points $A, B$ of maximum and minimum distance to $P$ (with respect to points on $S$ )

$$
\frac{\partial G(P, A)}{\partial \nu} \geqq \frac{\partial G(P, B)}{\partial \nu} ;
$$

equality implies that $S$ is a sphere with center $P$.

2. Mean values on hypersurfaces (continued). In this paragraph we generalize Theorem 1 to the case where the points $A, B$ may a priori lie on portions of $S$ which are not $C^{1+\alpha}$, in fact lie on $\sum \sum S_{i j}$ (recall that $S=\sum S_{i}$ $+\sum \sum S_{i j}$. We need a few preliminaries.

A point $Q$ on $S$ is called a convex angular point (with respect to $D$ ) if there exists a 2-plane $T$ passing through $Q$ and a ball $B_{\epsilon}$ with center $Q$ and radius $\epsilon$ such that the orthogonal projection of $D \cap B_{\epsilon}$ on $T$ is a domain $T_{0}$ whose boundary, in some neighborhood of $Q$ (in $T$ ), consists of two curves 
$l_{1}, l_{2}$ each having a uniformly continuous tangent, and forming an angle $<\pi$. It is further assumed that each point of $l_{1}, l_{2}$ is the projection of at least one point which belongs to the smooth portions of $S$, namely, to $\sum S_{i}$. $Q$ is a concave angular point (with respect to $D$ ) if it is a convex angular point with respect to the complement of $D+S$. Evidently, if $A(B)$ is an angular point, then it is a convex (concave) one.

For clarity, we now state some assumptions, needed in the statement of Theorem 2 below.

(A) If $A$ and $B$ lie on $\sum S_{i}$, then $S$ is $C^{1+\alpha}$ in some neighborhoods on $S$ of $A$ and $B ; \mu$ is continuous at $A, B$ and $\mu(A) \geqq \mu(B)$.

(B) If $A$ lies on $\sum \sum S_{i j}$, then $A$ is a convex angular point and, for some neighborhood $S_{0}$ on $S$ of $A, S_{0} \cap\left(\sum S_{i}\right)$ is $C^{1+\alpha}, \mu$ is continuous on $S_{0} \cap\left(\sum S_{i}\right)$ and for any sequence $\{Q\}$ converging to $A, Q \in S_{0} \cap\left(\sum S_{i}\right)$, $\lim \mu(Q) \neq 0$. A similar assumption is made with respect to $B$ (being a concave angular point), but with the requirement that $\lim \mu(Q) \neq \infty$.

We can now state the main result of the present section.

THEOREM 2. Let $\mu$ be an (absolutely) integrable function on $S$, and let $D$ be a Dirichlet domain. Finally, let the assumptions (A), (B) be satisfied. If the mean value property (1.1) holds for all harmonic polynomials, then $S$ is a sphere with center $P .\left(^{5}\right)$

Proof. If $A$ and $B$ lie on $\sum S_{i}$, then the assertion of the theorem follows by Theorem 1 . Hence, if we prove that $A$ and $B$ must lie on $\sum S_{i}$, then the proof of Theorem 2 is completed.

In view of the assumption (B) and Lemma 2, it suffices to establish the following two lemmas.

Lemma 3. If $A$ is a convex angular point, then $\partial G(P, Q) / \partial \nu \rightarrow 0$ for at least one sequence of points $Q \in \sum S_{i}, Q \rightarrow A$.

LemMA 4. If $B$ is a concave angular point, then $\partial G(P, Q) / \partial \nu \rightarrow-\infty$ for at least one sequence of points $Q \in \sum S_{i}, Q \rightarrow B$.

Proof of Lemma 3. The notation used in defining convex angularity will now be used. Accordingly, the orthogonal projection of $D \cap B_{\epsilon}(\epsilon>0$ fixed) on some 2-plane $T$ is a domain $T_{0}$. Near $A$, the boundary of $T_{0}$ consists of two curves $l_{1}, l_{2}$ having uniformly continuous first derivatives and forming at $A$ an angle $\beta<\pi$. For simplicity we may assume that $T$ is the 2-plane passing through the $x_{1}$ and $x_{2}$ axes and $A$ is the origin. We introduce a function $w=w\left(x_{1}, x_{2}\right)$ harmonic in $T_{0}$, continuous in the closure of $T_{0}$, which takes zero boundary values on $l_{1}+l_{2}$ and continuous positive boundary values on the remaining part of the boundary of $T_{0}$. We claim that

(5) See footnote in Theorem 1. 


$$
\frac{\partial w\left(x_{1}, x_{2}\right)}{\partial \nu_{0}} \rightarrow 0
$$

for a sequence $\left(x_{1}, x_{2}\right) \in l_{1}+l_{2}$ and $\rightarrow A$ where $\nu_{0}$ is the outward normal to $l_{1}+l_{2}$.

Proof of (2.1). Introducing the complex notation $z=x_{1}+i x_{2}$, we perform the conformal mapping $\zeta=z^{\pi / \beta}$. $T_{0}$ is mapped onto $\tilde{T}_{0}$ and $l_{1}+l_{2}$ onto a continuously differentiable curve $\tilde{l}=\tilde{l}_{1}+\tilde{l}_{2}$. The function $\tilde{w}(\zeta)=w(z)$ is harmonic in $T_{0}$. By a theorem of Warschawski [13] (see also [15]), it follows that $\partial w / \partial \nu_{0}$ and $\partial \tilde{w} / \partial \tilde{\nu}_{0}\left(\tilde{\nu}_{0}\right.$ is the outward normal to $\left.l\right)$ exist almost everywhere and satisfy

$$
\frac{\partial w}{\partial \nu_{0}}=\frac{\partial \tilde{w}}{\partial \tilde{\nu}_{0}}\left|\frac{d \zeta}{d z}\right| \quad \text { on } l_{1}+l_{2},
$$

and $\partial \tilde{w} / \partial \tilde{\nu}_{0}$ is in $L^{p}$ on $l_{1}+l_{2}$, for any $p>0$. Hence, any inequality of the form $\left|\partial \tilde{w} / \partial \tilde{\nu}_{0}\right| \geqq$ const $|z|^{-\delta}(\delta>0)$ cannot be satisfied almost everywhere on any subinterval of $l_{1}$ or $l_{2}$ beginning at $A$. Noting that $|d \zeta / d z|=\pi / \beta z^{8}$ where $\delta=(\pi-\beta) / \pi>0$, the proof of (2.1) follows.

We shall compare $w$ with $G$. We first introduce an appropriate domain. Consider the domain $C T_{0}$ defined as follows:

$C T_{0}=\left\{x=\left(x_{1}, \cdots, x_{n}\right) ;\left(x_{1}, x_{2}\right) \in T_{0},-\gamma<x_{i}<\gamma\right.$ for $\left.3 \leqq i \leqq n\right\}$.

We take $\gamma$ sufficiently large so that $D^{\prime} \equiv D \cap B_{\epsilon}$ is contained in $C T_{0}$. The sets $C l_{1}, C l_{2}$ are defined similarly to $C T_{0}$. The function $w$ constructed above, is harmonic also in $C T_{0}$, and it is positive in the closure of $C T_{0}$ except on $C l_{1}$ and $C l_{2}$ where it vanishes. Since $G(P, Q)$ vanishes on $S$, there exists a constant $K_{3}$ such that $K_{3} w>G$ on the boundary of $D^{\prime}$, and hence also throughout $D^{\prime}$.

Consider a sequence of points $Q$ which lie on the boundary of $D^{\prime}$ and whose orthogonal projection on $T$ lie on either $l_{1}$ or $l_{2}$ and for which (2.1) holds. We can take these points $Q$ to belong to the smooth portions of $S$. At such points $Q, K_{3} w=G=0$. Hence, at $Q$

$$
K_{3} \frac{\partial w}{\partial \nu} \leqq \frac{\partial G}{\partial \nu} \leqq 0 .
$$

If we denote by $\nu_{0}$ the outward normal to the boundary of $C T_{0}$ then, at the points $Q, \nu_{0}=\nu$ since the boundary of $C T_{0}$ is tangent at $Q$ to the boundary of $D^{\prime}$. Hence, at the points $Q$,

$$
K_{3} \frac{\partial w}{\partial \nu_{0}} \leqq \frac{\partial G}{\partial \nu} \leqq 0 .
$$

The left side of (2.2), however, tends to zero as $Q \rightarrow A$; hence the same is true for $\partial G / \partial \nu$.

The proof of Lemma 4 is similar to that of Lemma 3 and is therefore omitted. 
REMARK. All convex polyhedra (in fact, all convex hypersurfaces satisfying some mild differentiability assumptions) satisfy the assumptions of Theorem 2.

3. Application to conformal mappings. The assumption $\mu(A) \geqq \mu(B)$ made in Theorems 1, 2 is essential. Indeed, consider a 2-dimensional domain $D$ with a $C^{1+\alpha}$ boundary $S$, and let $w=f(z)$ map $D$ conformally and schlichtly onto $|w|<1$. Set $f\left(z_{0}\right)=0$. If $u(z)$ is harmonic in $D$, then $\tilde{u}(w)=u(z)$ is harmonic in $|w|<1$. The mean value theorem for harmonic functions $\tilde{u}(w)$ is

$$
\tilde{u}(0)=\frac{1}{2 \pi} \int_{|w|=1} \tilde{u} d S_{w} .
$$

Since $d S_{w}=\left|f^{\prime}(z)\right| d S_{z}$, we obtain

$$
u\left(z_{0}\right)=\int_{S} u(z) \frac{\left|f^{\prime}(z)\right|}{2 \pi} d S_{z}
$$

Hence, the mean value property (1.1) holds for $\mu(z)=\left|f^{\prime}(z)\right| / 2 \pi$, and $S$ is, in general, not a circle. The only condition in Theorem 1 which is violated, is $\mu(A) \geqq \mu(B)$.

From the above example we conclude that if $D$ is not a circle then $\left|f^{\prime}(A)\right|$ $<\left|f^{\prime}(B)\right|$. If $z_{0}=0$, and if $D$ is a circle, then $f(z)=C z, C=$ const and hence, $\left|f^{\prime}(A)\right|=\left|f^{\prime}(B)\right|$. We have thus proved (using a different notation):

THEOREM 3. Let $w=g(z)$ be a schlicht conformal mapping of $|z|<1$ onto a domain $D$ with a $C^{1+\alpha}$ boundary and let $g(z)$ be continuous on $|z| \leqq 1$. Let $z_{1}, z_{2}$ satisfy

$$
\max _{|z|=1}|g(z)|=\left|g\left(z_{1}\right)\right|, \quad \min _{|z|=1}|g(z)|=\left|g\left(z_{2}\right)\right|,
$$

and assume that $g(0)=0$. Then

$$
\left|g^{\prime}\left(z_{1}\right)\right| \geqq\left|g^{\prime}\left(z_{2}\right)\right|,
$$

and equality holds if and only if $f(z)=C z, C=$ constant.

Using [14], it follows that we can replace the assumption of $C^{1+\alpha}$ by the assumption that the tangent of the boundary of $D$ is a Dini continuous function of the length-parameter.

4. Mean values in solids. We shall maintain the notation of the previous sections, but consider now the mean value property with respect to solids, instead of hypersurfaces, that is,

$$
u(P)=\int_{D} u(Q) \mu(Q) d V_{Q}
$$

In the case of (1.1), there is only one density-function for which the mean value property holds (if $S$ is piecewise $C^{1+\alpha}$ ), namely, $-\partial G(P, Q) / \partial \nu$. In the 
present case this is no longer true. In fact, let $\gamma(Q)$ be any smooth function in $D+S$ satisfying the conditions

$$
\gamma=0 \text { on } S, \quad \frac{\partial \gamma}{\partial \nu}=-\frac{\partial G}{\partial \nu} \text { on } S .
$$

Then, using Green's formula and (1.1) with $\mu=-\partial G / \partial \nu$ we obtain the mean value property

$$
u(P)=\int_{D} u(Q) \Delta \gamma(Q) d V_{Q}
$$

Clearly, $\Delta \gamma$ can be any prescribed function in any given closed subset of $D$.

We shall first consider the case that $S$ is $C^{1+\alpha}$. (Then, $D$ is a Dirichlet domain.) We need auxiliary functions $\eta, \zeta$ defined as follows:

$\eta$ is the solution of

$$
\Delta \eta=\mu \text { in } D, \quad \eta=0 \text { on } S .
$$

From the general theory one knows that $\eta$ exists if $\mu$ is $C^{\alpha}$ in $D+S$, for some $\alpha>0 . \zeta$ is defined by

$$
\zeta(Q)=\zeta_{0}(Q)-\gamma_{n} \int_{D}^{2-n} r_{Q W}^{2-n} \frac{\partial \mu(W)}{\partial r} d V_{W},
$$

where $\partial / \partial r=\partial / \partial r_{P W}$ and where $\zeta_{0}$ is harmonic in $D$, continuous in $D+S$ and its boundary values are those which make $\zeta$ vanish on $S$. If $\mu$ is $C^{1+\alpha}$ in $D+S$, then

$$
\Delta \zeta=\frac{\partial \mu}{\partial r} \text { in } D-\{P\}, \quad \zeta=0 \text { on } S .
$$

Since $S$ is $C^{1+\alpha}$, one derives (by Kellogg [6]) the existence of $\eta \partial / \partial \nu$ and $\partial \zeta / \partial \nu$ on $S$.

We shall need the following assumptions:

(C) $\mu$ is $C^{1+\alpha}$ in $D+S$ and

$$
d(P, A) \mu(A)-d(P, B) \mu(B) \geqq \frac{\partial \zeta(A)}{\partial r}-\frac{\partial \zeta(B)}{\partial r} .
$$

(D) $\mu$ is $C^{\alpha}$ in $D+S$ and

$$
\frac{\partial \eta(A)}{\partial r} \geqq \frac{\partial \eta(B)}{\partial r} .
$$

We can now state the first result of the present section:

THEOREM 4. Let $S$ be $C^{1+\alpha}$ and iet (C) or (D) be satisfied. If the mean value property (4.1) holds for all harmonic polynomials, then $S$ is a sphere with center $P .{ }^{(5)}$ 
Proof. We first prove the theorem under the assumption (C).

We take a rectangular coordinate system $x=\left(x_{1}, \cdots, x_{n}\right)$ with $P$ for the origin and consider the mapping $y=\lambda x$, where $\lambda$ is a real number.

Let the image of $D$ be $D_{\lambda}$. Since $u(x / \lambda)$ is a harmonic polynomial whenever $u(x)$ is, we can write (4.1) for $u(x / \lambda)$. Then, using the substitution $y=\lambda x$, (4.1) becomes

$$
\lambda^{n} u(P)=\int_{D_{\lambda}} u(x) \mu(x / \lambda) d x
$$

Subtracting each side of (4.7) from the corresponding side in (4.1), then dividing by $\lambda-1$ and taking $\lambda \rightarrow 1$, we obtain

$$
n u(P)=\int_{S} u(Q) \mu(Q) h(Q) d S_{Q}-\int_{D} u(Q) \frac{\partial \mu(Q)}{\partial r_{P Q}} d V_{Q}
$$

where $h(Q)$ is a continuous function which depends linearly on the length of $P Q$ and which also depends on the angle between $P Q$ and the normal to $S$ at $Q$. In particular, recalling that $P Q$ is orthogonal to $S$ at $Q=A, Q=B$, we get

$$
h(A)=r_{P A}, \quad h(B)=r_{P B} .
$$

We now recall the definition of $\zeta$ and use Green's formula (which is easily justified since $\zeta$ has bounded first derivatives in $D-\{P\})$ to obtain, from (4.8),

$$
n u(P)=\int_{S} u(Q)\left[\mu(Q) h(Q)-\frac{\partial \zeta(Q)}{\partial \nu}\right] d S_{Q} .
$$

(4.10) has been proved for all harmonic polynomials. Hence, we can apply Theorem 1. Making use of (4.9), the proof of Theorem 4, under the assumption (C), is completed.

The proof under the assumption (D) is rather immediate. Indeed, using the definition of $\eta$ we obtain, by Green's formula,

$$
u(P)=\int_{S} u(Q) \frac{\partial \eta}{\partial \nu} d S_{Q}
$$

and the proof is completed by using Theorem 1 .

REMARK 1. Let $S$ be $C^{2}$. Using appropriate barriers and the definition of $\zeta$, one can show (see, for instance, $[10]$ ) that there exists a constant $K$ depending only on the diameter of $S$ and bounds on its first two derivatives, such that

$$
\max _{Q \in S}\left|\frac{\partial \zeta(Q)}{\partial \nu}\right| \leqq K\left|\frac{\partial \mu}{\partial r}\right|_{0}, \quad \text { where }\left|\frac{\partial \mu}{\partial r}\right|_{0}=\underset{W \in D}{\text { l.u.b. }}\left|\frac{\partial \mu(W)}{\partial r}\right|
$$


Hence the inequality in $(C)$ can be replaced by

$$
d(P, A) \mu(A)-d(P, B) \mu(B) \geqq 2 K\left|\frac{\partial \mu}{\partial r}\right|_{0}
$$

In particular, if $\partial \mu / \partial r \equiv 0,(4.11)$ becomes

$$
d(P, A) \mu(A) \geqq d(P, B) \mu(B) .
$$

We now extend Theorem 4 to the case of a piecewise smooth boundary $S$.

Theorem 5. Let $D$ be a Dirichlet domain, and let $A, B$ have some neighborhoods on $S$ which are $C^{1+\alpha}$. Let also one of the assumptions (C) or (D) be satisfied. If the mean value property (4.1) holds for all harmonic polynomials, then $S$ is a sphere with center $P .{ }^{(5)}$

Proof. It will be enough to establish the theorem under the assumption (C). We recall that throughout this paper $S$ is always assumed to be piecewise continuously differentiable. We also observe that $\zeta$ may be defined, and it exists, under our assumptions on $D$. Formula (4.8) can also be derived in a slightly different form, namely, there appear integrals on the $S_{i j}$. The main difficulty now is to be able to use Green's formula so as to derive (4.10).

To overcome this difficulty, we recall (compare the proof of Theorem 1), that once (4.8) has been established for all harmonic polynomials, its validity for any harmonic function in $D$, which is continuous in $D+S$, follows by approximation. Let $S_{0}$ be a $C^{1+\alpha}$ portion of $S$, and consider (4.8) only for nonnegative harmonic functions which are continuously differentiable in $D+S$ and which vanish outside a closed portion $S_{1}$ lying in the interior of $S_{0}$, and, finally, which are harmonic also across $S_{1}$, i.e., in some $n$-dimensional neighborhood of $S_{1}$. Let $S_{2}^{e}$ be the equi-potential hypersurface $u=\epsilon$ in $D+S$, considered for such $\epsilon>0$ for which the gradient of $u$ does not vanish at any of its points. Although $S_{2}^{\epsilon}$ intersects $S_{1}$, still for almost all $\epsilon$ the above property (concerning gradient $u$ ) holds since $u$ is assumed to be harmonic in some $n$ dimensional neighborhood of $S_{1}$. Let $D^{\epsilon}$ be the subset of $D$ defined by $u>\epsilon$, and let $S_{1}^{e}$ be that part of the boundary of $D^{\epsilon}$ which lies on $S$. Then, applying Green's formula in $D^{\epsilon}$, we get

$$
\begin{aligned}
-\int_{D \epsilon} u(Q) \frac{\partial \mu(Q)}{\partial r_{P Q}} d V_{Q}= & \int_{S^{\epsilon}} \zeta(Q) \frac{\partial u(Q)}{\partial \nu} d S_{Q} \\
& -\int_{S_{1}^{\epsilon}} u(Q) \frac{\partial \zeta(Q)}{\partial \nu} d S_{Q}-\int_{S_{2}^{e}} u(Q) \frac{\partial \zeta(Q)}{\partial \nu} d S_{Q}
\end{aligned}
$$

where $S^{\epsilon}=S_{1}^{\epsilon}+S_{2}^{\epsilon}$.

The first integral on the right side of (4.13) tends to zero as $\epsilon \rightarrow 0$, since $\zeta=0$ on $S_{1}^{\epsilon}, \zeta \rightarrow 0$ on $S_{2}^{\epsilon}$ as $\epsilon \rightarrow 0$, and $\int_{S_{2}^{\epsilon}}^{\epsilon}|\partial u / \partial \nu| d S=\int_{S_{2}^{\epsilon}}^{\epsilon}(-\partial u / \partial \nu) d S$ $=\int_{S_{1}^{\epsilon}}(\partial u / \partial \nu) d S$ is bounded. Since $\zeta$ is continuously differentiable in some portion of $D+S$ neighboring $S_{0}$, the second integral tends to 


$$
\int_{S_{1}} u(Q) \frac{\partial \zeta(Q)}{\partial \nu} d S_{Q}
$$

Finally, the third integral on the right side of (4.13) is equal to

$$
\epsilon \int_{S_{2}} \frac{\partial \zeta}{\partial \nu} d S=\epsilon \int_{S^{\epsilon}} \frac{\partial \zeta}{\partial \nu} d S-\epsilon \int_{S_{1}^{\epsilon}} \frac{\partial \zeta}{\partial \nu} d s=\epsilon \int_{D^{\epsilon}} \Delta \zeta d V-\epsilon \int_{S_{1}} \frac{\partial \zeta}{\partial \nu} d S
$$

Each of the last two integrals is bounded independently of $\epsilon$ (recall that $\Delta \zeta=\partial \mu / \partial r)$. Hence, the third integral on the right side of (4.13) tends to zero as $\epsilon \rightarrow 0$. Taking $\epsilon \rightarrow 0$ in (4.13) and using the result thus obtained in (4.8), we obtain (4.10).

In order to establish (4.10) for any continuous boundary values of $u$ on $S_{1}, u=0$ on $S-S_{1}$, we use approximation (as in the proof of Theorem 1 ).

Having proved (4.10) we can now complete the proof of Theorem 4 by applying Theorem 1 .

REMARK 1. Theorem 4 is the analog of Theorem 1 to the case of mean values in solids. Clearly, Theorem 2 can also be extended analogously. Details will be omitted.

REMARK 2. Let $\sigma$ be a union of nonoverlapping surfaces, each being smooth and of dimension $\leqq n-2$. Let $S$ be piecewise continuously differentiable hypersurface on which $\sigma$ lies and suppose that the points $A, B$ have neighborhoods on $S$ which are $C^{1+\alpha}$. If the mean value property

$$
u(P)=\int_{\sigma} u(Q) \mu(Q) d \sigma_{Q}
$$

holds for all harmonic polynomials, then by the proof of Theorem 1 (with $\mu(A)=\mu(B)=0$; since we can always choose $S$ such that $A, B$ do not belong to $\sigma$ ) it follows that $S$ is a sphere with center $P$. But then,

$$
u(P)=\beta \int_{S} u(Q) d S_{Q} \quad(\beta \text { appropriate constant })
$$

which is clearly in contradiction to (4.14). Hence:

There exists at least one harmonic polynomial which does not satisfy the mean value property (4.14).

REMARK 3. Let $M$ be the distribution corresponding to the density $\mu$ on $S$, on $D$, or on $\sigma$. According to Choquet and Deny (see [11, p. 73]), the mean value property for all harmonic polynomials is satisfied if and only if there exists a distribution $L$ with a compact support such that

$$
\Delta L=\delta-M, \quad \delta=\text { Dirac measure. }
$$

Our results can therefore be stated in terms of solutions of the differential equation (4.15). 
5. Mean values for parabolic solutions. In this section we shall extend the previous results to the case of parabolic (or caloric) functions i.e., functions satisfying the heat equation $\Delta u=\partial u / \partial t$. We introduce the notation:

$$
C D=\{(Q, t) ; Q \in D, 0<t<\tau\}, \quad C S=\{(Q, t) ; Q \in S, 0<t<\tau\}
$$

where $\tau>0$ is fixed, and denote by $G(P, \tau ; Q, t)$ the Green's function of the equation $\Delta u+\partial u / \partial t=0$ in $C D$, having its singularity at the fixed point $(P, \tau)$. Since the analog of Kellogg's result [6] has not appeared so far in the literature, we shall have to replace the $C^{1+\alpha}$ assumption of the previous sections by a $C^{2+\alpha}$ assumption, in which case a solution which assumes a $C^{2+\alpha}$ data (on the $C^{2+\alpha}$ portion of $C S$ ) is $C^{2+\alpha}$ in some one-sided neighborhood of that portion lying in $[C D]^{-}$(the closure of $C D$ ); see [2].

We recall the fact [12] that $D$ is a Dirichlet domain for $\Delta u=0$ if and only if $C D$ is a Dirichlet domain for $\Delta u=u_{t}$.

We shall assume a mean value property of the form

$$
u(P, \tau)=\int_{0}^{\tau} \int_{S} u(Q, t) \mu(Q, t) d S_{Q} d t
$$

for solutions of

$$
\Delta u=u_{t} \text { in } C D, \quad u=0 \text { on } D .
$$

It may be recalled that, under some smoothness assumptions on $S$ (for instance if $S$ is $\left.C^{2+\alpha}\right),(5.1)$ is satisfied with $\mu=-\partial G / \partial \nu$. If $S$ is a sphere with center $P$, then by reason of symmetry one concludes that

$$
-\frac{\partial G(P, \tau ; Q, t)}{\partial \nu}=\beta(t)
$$

where $\beta(t)$ is some non-negative function (and in fact, positive for $0 \leqq t<\tau$; by $[3])$.

In analogy with Theorem 1 we shall prove:

THEOREм 6. Let $\mu$ be an (absolutely) integrable function on CS, continuous at the points $(A, t),(B, t)$ for all $\tau-\delta<t<\tau$ (for some $\delta>0, \delta \leqq \tau$ ) and let $\mu(A, t) \geqq \mu(B, t)$. Let $D$ be a Dirichlet domain and let $S$ be $C^{2+\alpha}($ for some $\alpha>0)$ in some neighborhoods of the points $A, B$. If the mean value property (5.1) holds for all solutions of (5.2) which is continuous in $[C D]^{-}$, then $S$ is a sphere with center $P$.

Proof. Following the proof of Theorem 1, we first wish to establish an analog of Lemma 1.

LEMMA 5. If $u$ is a solution of (5.2), continuous in [CD]- and vanishes outside $S_{1} \times\left[t_{1}, t_{2}\right]$ where $0<t_{1}<t_{2}<\tau$ and $S_{1}$ is a closed portion of $S$ contained in an open $C^{2+\alpha}$ portion $S_{0}$ of $S$, then 


$$
u(P, \tau)=-\int_{t_{1}}^{t_{2}} \int_{S_{1}} \frac{\partial G(P, \tau ; Q, t)}{\partial \nu} u(Q, t) d S_{Q} d t
$$

where $G(P, \tau ; Q, t)$ is Green's function of $u+u_{t}=0$ in $C D$, having $(P, \tau)$ for its pole.

Proof. By [3], $\partial G / \partial \nu \neq 0$ on $S_{1} \times\left[t_{1}, t_{2}\right]$. Hence, in some one-sided neighborhood $\Sigma_{1}$ of $S_{1} \times\left[t_{1}, t_{2}\right]$ lying in [CD]- and below $t=\tau$, the gradient of $G(P, \tau ; Q, t)$ is not zero. Thus, the hypersurfaces $G=\epsilon$ intersect $\Sigma_{1}$ in some continuously differentiable portions $\sigma_{\epsilon}$. We now construct a domain $C^{\epsilon} D$ having the following properties:

(i) It lies in $0<t<t_{3}$ for some $t_{2}<t_{3}<\tau$ and has $t=0, t=t_{3}$ for parts of its boundary; denote them by ' $D_{0},{ }^{'} D_{3}$.

(ii) It lies in $C D$, and its boundary in $0<t<t_{3}$ consists of a continuously differentiable hypersurface which is the disjoint union of $\sigma_{\epsilon}$ and some $\tilde{\sigma}_{\epsilon}, \tilde{\sigma}_{\epsilon}$ lying outside $\Sigma_{1}$.

(iii) As $\epsilon \rightarrow 0$, the points of $\tilde{\sigma}_{\epsilon}$ (and clearly also those of $\sigma_{\epsilon}$ ) tend to the boundary $C S$ of $C D$.

In the domain $C^{\epsilon} D$ we can apply Green's formula, thus obtaining

$$
\begin{aligned}
\int_{\Phi_{D_{3}}} u\left(Q, t_{3}\right) G\left(P, \tau ; Q, t_{3}\right) d V_{Q}= & -\int_{\sigma_{\epsilon}} u \frac{\partial G}{\partial \nu} d \sigma-\int_{\tilde{\sigma}_{\epsilon}} u \frac{\partial G}{\partial \nu} d \sigma \\
& +\int_{\sigma_{\epsilon}} G \frac{\partial u}{\partial \nu} d \sigma+\int_{\tilde{\sigma}_{\epsilon}} G \frac{\partial u}{\partial \nu} d \sigma
\end{aligned}
$$

It will be enough to consider the case $n>1$, since in the case $n=1$ Green's function is known explicitly and the proof of the lemma follows by a straightforward calculation which shows that the right side of (5.3) is a solution of (5.2) with the boundary values of $u$ on $C S$.

Now, using the interior estimates of [1] we know that

$$
\frac{\partial G}{\partial \nu}=O\left(\frac{1}{d}\right), \quad \frac{\partial u}{\partial \nu}=O\left(\frac{1}{d}\right),
$$

where $d$ is the distance from the point to $C S$. Since $u \rightarrow 0$ on $\tilde{\sigma}_{\mathrm{e}}$ and $G \rightarrow 0$ on $\sigma_{\epsilon}+\tilde{\sigma}_{\epsilon}$, as $\epsilon \rightarrow 0$, we conclude, if $n>1$, that the last three integrals on the right side of (5.4) tend to zero as $\epsilon \rightarrow 0$.

To study the behavior of the first integral, we construct a domain $\Sigma_{\text {e }}$ bounded by a portion $\sigma_{0 \epsilon}$ on $C S$ containing $S_{1} \times\left[t_{1}, t_{2}\right]$, by $\sigma_{\epsilon}$ and by the lateral boundary of a cylinder having $\sigma_{\epsilon}, \sigma_{0 \epsilon}$ for bases. $\Sigma_{\epsilon}$ can be constructed similarly to the construction of the cylinder $V^{\epsilon}$ in Lemma 1 . (We may assume, without restricting the generality of the proof, that the diameter of $S_{0}$ is sufficiently small.) Then, using Green's formula in $\Sigma \epsilon$, it follows that, as $\epsilon \rightarrow 0$, the first integral on the right side of (5.4) tends to 


$$
\int_{t_{1}}^{t_{2}} \int_{S_{1}} u \frac{\partial G}{\partial \nu} d S d t
$$

Taking $\epsilon \rightarrow 0$ in (5.4), and then taking, in the equation obtained, $t_{3} \rightarrow \tau$, (5.3) follows.

Combining Lemma 5 with the property (5.1) we obtain an analog of Lemma 2. In particular it follows that

$$
-\frac{\partial G(P, \tau ; Q, t)}{\partial \nu}=\mu(Q, t) \quad \text { for } Q=A, B \text { and } \tau-\delta<t<\tau .
$$

To complete the proof of Theorem 6 , we have to compare $\partial G / \partial \nu$ at the points $(A, t),(B, t)$ using the geometric definition of $A$ and $B$.

Green's function can be written in the form

$$
G(P, \tau ; Q, t)=K_{n}(\tau-t)^{-n / 2} \exp \left\{-\frac{d(P, Q)^{2}}{4(\tau-t)}\right\}+h(Q, t),
$$

where $K_{n}$ is some positive constant, and $h(Q, t)=0, Q \in D$.

We now introduce (as in $\$ 1$ ) a ball $K_{A}$ with center $P$ and radius $r_{P A}$, whose boundary is denoted by $S_{A}$. Let $C K_{A}$ be the cylinder $K_{A} \times(0, \tau)$ and denote by $G_{A}(P, \tau ; Q, t)$ the Green's function of $\Delta u+u_{t}=0$ in $C K_{A}$, having its singularity at $(P, \tau)$. We then write, in analog with (5.6),

$$
G_{A}(P, \tau ; Q, t)=K_{n}(\tau-t)^{-n / 2} \exp \left\{-\frac{d(P, Q)^{2}}{4(\tau-t)}\right\}+h_{A}(Q, t) .
$$

It is easy to see that $G_{A}>0$ in $C K_{A}$. Indeed, for any solution $v$ of the heat equation in $C K_{A}$ which vanishes on $C S$ we have:

$$
v(P, \tau)=\int_{D} G_{A}(P, \tau ; Q, t) v(Q, t) d V_{Q} .
$$

If $v\left(Q, t_{0}\right) \geqq 0$ and $\not \equiv 0$ for $Q \in D$, and if $t_{0}$ is fixed $\left(0<t_{0}<\tau\right)$, then by the maximum principle [9], $v(P, \tau)>0$. From this it follows that $G_{A}>0$ in $C K_{A}$. Hence, applying once more the maximum principle to $G_{A}-G$ we conclude that $G_{A}-G>0$ in $C D$. Since at $Q=A, G_{A}=G$, we obtain the inequality

$$
\frac{\partial G_{A}(P, \tau ; Q, t)}{\partial \nu} \leqq \frac{\partial G(P, \tau ; Q, t)}{\partial \nu} \quad \text { at } Q=A .
$$

Next it can be shown that

$$
\begin{aligned}
\frac{\partial}{\partial \nu} G_{A}(P, \tau ; Q, t)= & {\left[\frac{\partial}{\partial \nu}\left[K_{n}(\tau-t)^{-n / 2} \exp \left\{-\frac{(d(P, Q))^{2}}{4(\tau-t)}\right\}\right]\right] } \\
& \cdot\left(1+O\left(\exp \left\{-\frac{\rho}{\tau-t}\right\}\right)\right)
\end{aligned}
$$

for some $\rho>0$. 
Indeed, let $G_{0}$ be Green's function in the cylinder with a base which is an $n$-dimensional cube containing $K_{A}$ and tangent to it at the point $Q$. Then $G_{0}-G_{A}>0$ in $C K_{A}$. Writing $G_{0}$ in the form (5.6) with $h=h_{0}$ we then have $h_{0}-h_{A}=G_{0}-G_{A}>0$ in $C K_{A}$, and $h_{0}-h_{A}=0$ at $(Q, t)$. Hence

$$
\frac{\partial h_{0}}{\partial \nu} \leqq \frac{\partial h_{A}}{\partial \nu} .
$$

On the other hand, $h_{A}$ on $S_{A} \times\left(t_{0}, \tau\right)$ is a monotone increasing function of $t$ if $t_{0}$ is sufficiently close to $\tau$. Hence $\partial h_{A} / \partial t>0$ on $S_{A} \times\left(t_{0}, \tau\right)$. On $t=\tau, \partial h_{A} / \partial t$ $=-\Delta h_{A}=0$. Using the maximum principle we conclude that $\partial h_{A} / \partial t>0$ in $K_{A} \times\left(t_{0}, \tau\right)$. Hence, $\Delta h_{A}=-\partial h_{A} / \partial t<0$. By the maximum principle (for subharmonic functions) $h_{A}(W, t)$ attains its minimum in $W \in K_{A}+S_{A}$ ( $t$ fixed) on $S_{A}$. Since, however, $h_{A}(W, t)=$ const (depending on $t$ ) for $W \in S_{A}$, we get

$$
\frac{\partial h_{A}}{\partial \nu} \leqq 0 .
$$

Now, $G_{0}$ can be written explicitly in a rather simple manner using the method of images (see, for instance, $[4$, p. 514]). It is then immediately seen that (5.9), with $G_{A}$ replaced by $G_{0}$, is valid, i.e., $\partial h_{0} / \partial \nu$ is bounded by the error term of (5.9). Combining this with the inequalities (5.10), (5.11), the proof of (5.9) is completed.

Inequalities similar to (5.8), (5.9), hold also for $G_{B}$, which is defined with respect to $K_{B}$. Combining these inequalities with (5.8), (5.9), then using (5.5) and the assumption $\mu(A, t) \geqq \mu(B, t)$ we get

$$
\begin{array}{r}
{\left[\frac{K_{n}}{2}(\tau-t)^{-(n+1) / 2} d(P, B) \exp \left\{-\frac{(d(P, B))^{2}}{4(\tau-t)}\right\}\right]} \\
\cdot\left(1+O\left(\exp \left\{-\frac{\rho}{\tau-t}\right\}\right)\right) \\
\leqq\left[\frac{K_{n}}{2}(\tau-t)^{-(n+1) / 2} d(P, A) \exp \left\{-\frac{(d(P, A))^{2}}{4(\tau-t)}\right\}\right] \\
\cdot\left(1+O\left(\exp \left\{-\frac{\rho}{\tau-t}\right\}\right)\right) .
\end{array}
$$

If $d(P, A)>d(P, B)$ then the brackets on the right side of the inequality become smaller than those on the left side of the inequality if $t$ is sufficiently close to $\tau$ (in fact if $\left.2(\tau-t)<(d(B, P))^{2}\right)$. Hence, taking $t \rightarrow \tau$ in (5.12) we derive a contradiction, which proves that $d(P, A)=d(P, B)$, i.e., $S$ is a sphere with center $P$.

REMARK 1. Theorems $2,4,5$ can also be generalized in the parabolic case. We mention briefly an extension of Theorem 2. The main point is the generalization of Lemmas 3, 4. More specifically, we have to construct a com- 
parison function analogous to $w\left(x_{1}, x_{2}\right)$. The new function must satisfy the equation $\Delta u+u_{t}=0$. But for such a function one may take the very same harmonic function $w\left(x_{1}, x_{2}\right)$. The domain of comparison is now a cylinder $T_{0} \times(0, \tau)$. On the lateral side of this cylinder $w$ majorizes Green's function, as in $\S 2$. At the top $t=\tau, w \geqq 0$, whereas $G=0$. Hence the proof of Lemma 3 can be carried out, for the parabolic case, in a similar manner. We conclude that the analog of Theorem 2 holds in the parabolic case. Theorems 4, 5 can also be generalized without any difficulty.

REMARK 2. One may replace (5.1) by

$$
u(P, \tau)=\int_{0}^{\tau} \int_{S} u(Q, t) \mu(Q, t) d S_{Q} d t+\int_{D} u(Q, 0) \lambda(Q) d V_{Q}
$$

for all solutions of $\Delta u=u_{t}$, where $\lambda$ is an integrable function. Then Theorem 6 remains true if one assumes that $D$ is convex and that (5.13) holds for all polynomial solutions of the heat equation. Indeed, using an approximation theorem of Malgrange [8] one concludes that (5.13) is satisfied for any parabolic solution in a neighborhood of $[C D]^{-}$. Then, by approximating $D$ by domains from the outside, one derives (5.13) for all parabolic solutions in $C D$, continuous in $[C D]^{-}$. In particular, for solutions of $(5.2),(5.1)$ is satisfied, so that the assertion of Theorem 6 is valid.

6. Mean values for hyperbolic solutions. We shall discuss the hyperbolic case very briefly. We shall consider mean value properties for solutions of the wave equation in various domains.

CASE I. Let $K$ be a conical surface with base $D$ lying on $t=0$ and vertex $(P, \tau), \tau>0$, and let $S$ be the boundary of $D$. We consider the mean value property

$$
u(P, \tau)=\int_{D} u(Q, 0) \mu(Q) d V_{Q}+\int_{S} u(Q, 0) \lambda(Q) d S_{Q} \quad(\mu \neq 0)
$$

for all solutions of

$$
\Delta u=u_{t t}, \quad u_{t}(Q, 0)=0 .
$$

We claim that if (6.1) holds for all solutions of (6.2) ( $Q$ varies in the $n$-dimensional Euclidean space $E$ and $0<t<\tau)$, then $S$ is a sphere with center $P$.

Indeed, if $n$ is odd then we know that

$$
u(P, \tau)=\int_{r_{P Q}=\tau} u(Q, 0) \tilde{\lambda}\left(r_{P Q}\right) d V_{Q}
$$

and if $n$ is even then we know that

$$
u(P, \tau)=\int_{r_{P Q}<\tau} u(Q, 0) \hat{\mu}\left(r_{P Q}\right) d S_{Q}
$$


where $\tilde{\lambda}>0, \tilde{\mu}>0$. Since the initial values of $u(P, 0)$ can be taken in an arbitrary manner, it follows that $S$ is the sphere $r_{P Q}=\tau$.

CASE II. Let $\hat{K}$ be the lateral boundary of the cone $K$ considered above, and let $n$ be odd. Imitating Huygens' principle we consider a mean value property of the form

$$
u(P, \tau)=\int_{\hat{K}} u(Q, t) \hat{\mu}(Q, t) d \hat{K}+\int_{\hat{K}} \frac{\partial u(Q, t)}{\partial t} \tilde{\lambda}(Q, t) d \hat{K} .
$$

We claim that if (6.5) is satisfied for all solutions of the wave equation in $(Q, t)(Q \in E, 0<t<\tau)$, then $\hat{K}$ is a circular cone.

The proof is an immediate consequence of Huygens' principle which states that $u(P, \tau)$ depends only on the values of $u$ and $\partial u / \partial t$ at points $(Q, t)$ which satisfy the condition: $r_{P Q}=\tau-t$.

CASE III. Consider in the plane $(n=1)$ the mean value property

$$
u\left(x_{0}, t_{0}\right)=\sum_{i=1}^{N} \mu_{i} u\left(x_{i}, \tau_{i}\right)
$$

for all solutions of $u_{x x}=u_{t t}$. As is well known, any solution satisfies the property:

$$
u(x, y)=u(x, b)+u(a, y)-u(a, b)
$$

for any pair of points $(a, b),(x, y)$. It can now be easily shown, that if (6.6) holds then the points $\left(x_{i}, \tau_{i}\right)$ can be divided into disjoint sets each consisting of four points of the form

$$
(x, y),(x, b),(a, y),(a, b)
$$

for some $(x, y)$, and $(a, b)=\left(x_{0}, t_{0}\right)$.

\section{REFERENCES}

0. W. Brödel, Funktionen mit Gaussischer Mittelwerteigenschaft für konvexe Kurven und Bereiche, Deutsche Math. vol. 4 (1939) pp. 3-15.

1. A. Friedman, Interior estimates for parabolic systems of partial differential equations, J. Math. Mech. vol. 7 (1958) pp. 393-417.

2. - Boundary estimates for second order parabolic equations and their applications, J. Math. Mech. vol. 7 (1958) pp. 771-791.

3. - Remarks on the maximum principle for parabolic equations and its applications, Pacific J. Math. vol. 8 (1958) pp. 201-211.

4. - Parabolic equations of the second order, Trans. Amer. Math. Soc. vol. 93 (1959) pp. 509-530.

5. O. D. Kellogg, Singular manifolds among those of an analytic family, Bull. Amer. Math. Soc. vol. 35 (1929) pp. 711-718.

6. - On the derivatives of harmonic functions on the boundary, Trans. Amer. Math. Soc. vol. 33 (1931) pp. 486-510.

7. - Foundations of potential theory, New York, Dover Publications, 1929. 
8. B. Malgrange, Existence et approximation des solutions des équations aux dérivées partielles et des equations de convolution, Ann. Inst. Fourier, Grenoble vol. 6 (1955-1956) pp. 271-354.

9. I. Nirenberg, A strong maximum principle for parabolic equations, Comm. Pure Appl. Math. vol. 6 (1953) pp. 167-177.

10. - Existence theorems in partial differential equations, New York University, Notes.

11. L. Schwartz, Theorie des distributions. II, Paris, Hermann, 1959.

12. A. Tychonoff, Sur l'équation de la chaleur de plusieurs variables, Bull. l'Univ. d'Etat Moscow vol. 1 (1938).

13. S. E. Warschawski, Über einige Konvergenzsätze aus der Theorie der konformen $A b$ bildung, Nachr. Ges. Wiss. Göttingen (1930) pp. 344-369.

14. - Über das Randverhalten der Ableitung der Abbildungsfunktion bei konformer Abbildung, Math. Z. vol. 35 (1932) pp. 321-446.

15. - On conformal mapping of regions bounded by smooth curves, Proc. Amer. Math. Soc. vol. 2 (1951) pp. 254-261.

16. M. Brelot, Sur l'approximation et la convergence dans la theorie des fonctions harmoniques, Bull. Soc. Math. France vol. 73 (1945) pp. 55-70.

University of Minnesota, Institute of TechNology, Minneapolis, Minnesota 